Ciência Florestal, Santa Maria, v. 23, n. 3, p. 483-488, jul.-set., 2013 ISSN 0103-9954

\title{
PASTAS ESTIMULANTES EM SISTEMAS DE RESINAGEM DE Pinus elliottii var. elliottii
}

\author{
STIMULATING PASTES ON SYSTEM FOR RESIN FLOW OF Pinus elliottii var. elliottii
}

\author{
André Luiz Marretto Fusatto ${ }^{1}$ Paulo Roberto de Camargo e Castro ${ }^{2}$ Stella Consorte Cato ${ }^{3}$ \\ José Otávio Brito ${ }^{4}$
}

\section{RESUMO}

A utilização de pastas estimulantes tem por finalidade aumentar a taxa de fluxo de resina e o seu tempo de duração. No Horto Florestal de Itatinga - SP, 159 árvores de Pinus elliottii var. elliottii foram submetidas a tratamentos com 5 pastas estimulantes, Pasta Vermelha Alchem, Pasta com Ethephon Planebrás, Pasta Preta Resinas Brasil, Pasta Vermelha Eldorado e Pasta Teste (Paraquat + Ethephon) sendo comparadas ao Controle (Pasta Preta Planebrás). Foram realizadas explotações quinzenais e coletas trimestrais (do resultado de 5 estrias), determinando-se o peso de resina das árvores durante 2,5 anos. A aplicação de pastas estimulantes com ethephon não mostrou vantagem sobre aplicação de pastas sem ethephon. As pastas estimulantes aplicadas diferiram nas pesagens realizadas. Nos tratamentos efetuados, a porcentagem de breu variou de $71,33 \%$ a $76,56 \%$ e a terebintina entre $10,37 \%$ a $13,78 \%$. O teor de alfa-pineno variou de $37,70 \%$ a $53,31 \%$, sendo que o de beta-pineno de $34,34 \%$ a $50,41 \%$.

Palavras-chave: Pinus; estimulação; resina.

\begin{abstract}
S
The use of stimulating pastes aims to increase the rate of resin flow and its time of duration. In the Forest Experiment Station of Itatinga, São Paulo state, 159 trees of Pinus elliottii var. elliottii were were submitted to the treatments with 5 stimulant pastes, (Red Alchem, Paste with Ethephon Planebrás, Black Resin Brazil, Red Eldorado and Test Paste (Paraquat + Ethephon) that was compared to the Control (Black Planebrás). Biweekly chipping and collects every three months were accomplished (from the result of 5 grooves), being determined the resin weight during 2.5 years. The application of stimulating pastes with ethephon did not show significant advantage about application of pastes without ethephon. The stimulant pastes applied were different in the weightings performed. In the treatments applied, the percentage of pitch ranged from $71.33 \%$ to $76.56 \%$ and the turpentine ranged from $10.37 \%$ to $13.78 \%$. The yield of alpha-pinene ranged from $37.70 \%$ to $53.31 \%$, and of beta-pinene from $34.34 \%$ to $50.41 \%$.
\end{abstract}

Keywords: Pinus; stimulation;resin.

\section{INTRODUÇÃO}

A indústria de resina envolve duas etapas de trabalho, a que se desenvolve em condições de campo e o sistema industrial. A primeira tem por finalidade a extração da resina das árvores e a segunda desdobrar a resina em seus constituintes de uso comercial (BUCCI et al., 1986).
$\mathrm{O}$ sistema de resinagem convencional envolve a seleção de árvores com base na média de produção de resina. Primeiramente, deve-se descorticar as árvores 15 a 20 dias antes da resinagem, e então, procede-se a marcação do comprimento do painel, correspondente a $1 / 3$ do perímetro do tronco. Coloca-se o recipiente de coleta $20 \mathrm{~cm}$ acima do solo. Efetua-se a abertura do painel de resinagem

1 Engenheiro Agrônomo, MSc., Departamento de Ciências Biológicas, Escola Superior de Agricultura Luiz de Queiróz, Universidade de São Paulo, Av. Pádua Dias, 11, CEP 13418-900, Piracicaba (SP).

2 Engenheiro Agrônomo, Professor Titular do Departamento de Ciências Biológicas, Escola Superior de Agricultura Luiz de Queiróz, Universidade de São Paulo, Av. Pádua Dias, 11, CEP 13418-900, Piracicaba (SP).prcastro@usp.br

3 Engenheira Agrônoma, Dr., Assessora de Pesquisa da Stoller do Brasil, Caixa Postal 55, CEP 13150-000, Cosmópolis (SP).

4 Engenheiro Florestal, Professor Titular do Departamento de Ciências Florestais, Escola Superior de Agricultura Luiz de Queiróz, Universidade de São Paulo, Av. Pádua Dias, 11, CEP 13418-900, Piracicaba (SP).

Recebido para publicação em 31/10/2011 e aceito em 2/05/2012 
com execução de corte de 18 a $22 \mathrm{~cm}$, com $2 \mathrm{~cm}$ de largura, para a aplicação da pasta (CAPITANI et al, 1980).

Desde o início da exploração resinífera no Brasil, o ácido sulfúrico vem sendo usado como estimulante. No entanto, a procura de novas formulações de pasta estimulante é contínua e é neste contexto que o ethephon tem surgido como um produto adicional (GARRIDO, 1983).

Ethephon é adicionado nas pastas vermelhas em teores de cerca de 5\% de acordo com Peters e Roberts (1977), Joyce e Drew (1979), Capitani (1982), Garrido (1983), Bucci et al. (1986) e Fernandes e Osório (1988). Além disso, essas pastas contêm material inerte e água ou óleo como solvente, conforme Pardos (1980) e Capitani (1982).

Para que haja exsudação, é necessário cortar os canais resiníferos para acelerar o processo de formação e secreção de resina. Acredita-se que, tanto a lesão promovida pela estria, como a lesão química, provocada pela aplicação de pastas estimulantes, estejam relacionadas à síntese de etileno, sendo que este hormônio vegetal encontra-se envolvido no processo de ativação do fluxo de resina (CAPITANI, 1982). Sugere-se que o etileno deve ser um dos fatores de iniciação da síntese de resina a partir das células epiteliais internas das cavidades resiníferas.

Podem-se estabelecer intervalos de 12 a 15 dias para aplicação da pasta, com o objetivo de se obter dados comparáveis. Árvores tratadas com pasta rendem de 30 a $40 \%$ a mais de resina. Podem-se estabelecer intervalos de quatro semanas para a coleta. De acordo com Alvim (1969), foi determinado um esquema em que os fatores ambientais mais relevantes (radiação, temperatura, água e nutrição) interferem na produção de resina de Pinus.

Com o objetivo de estabelecer a eficiência, homogeneidade e características tecnológicas obtidas com o uso de diferentes pastas para resinagem de Pinus elliottii var. elliottii, um experimento foi desenvolvido no Horto Florestal de Itatinga (SP), em um povoamento de Pinus elliottii var. elliottii.

\section{MATERIAL E MÉTODO}

O experimento foi instalado em um povoamento homogêneo de Pinus elliottii var. elliottii, localizado no Horto Florestal de Itatinga (SP).

Inicialmente, para a seleção das árvores que viriam a compor os tratamentos, a área experimental foi dividida em 6 blocos contendo 100 árvores cada.
Foram realizadas duas coletas, correspondentes à produção de 5 estrias, e a resina foi pesada para a determinação das produções das 100 árvores de cada bloco, obtidas com a estimulação originalmente por pasta vermelha. Com base na média de produção de resina por árvore e nos desvios padrões da média, foram selecionadas, em cada bloco, as árvores que compuseram o experimento.

Foram realizadas coletas e pesagens de resina para obtenção de produções individuais de resina nas 600 árvores de Pinus elliottii var. elliottii distribuídas nos 6 talhões.

Árvores com produção de resina dentro de maiores frequências no interior de cada bloco, também foram selecionadas.

Iniciaram-se os tratamentos com cinco diferentes pastas comerciais de estimulação e uma pasta teste elaborada com $100 \mathrm{~g}$ Paraquat / $\mathrm{kg}$ pasta, $450 \mathrm{~g}$ Ethephon / kg pasta, $200 \mathrm{~g} / \mathrm{kg}$ Farelo de Arroz / kg pasta e Água. Nessas árvores selecionadas, foi feita uma abertura da face a $50 \mathrm{~cm}$ de altura.

Os tratamentos realizados foram - Controle: Pasta Planebrás; Pasta A: Pasta Vermelha Alchem; Pasta B: Pasta com Ethephon Planebrás; Pasta C: Pasta Preta Resinas Brasil; Pasta D: Pasta Vermelha Eldorado e Pasta Teste: Paraquat + Ethephon.

A produção de resina foi estabelecida em coletas realizadas a cada 3 meses, nas árvores selecionadas, sendo que as mesmas foram efetuadas durante 2,5 anos.

Ethephon é adicionado nas pastas vermelhas em teores de cerca de $5 \%$ de acordo com Peters e Roberts (1977), Joyce e Drew (1979), Capitani (1982), Garrido (1983), Bucci et al. (1986) e Fernandes e Osório (1988). Além disso, essas pastas contêm material inerte e água ou óleo como solvente, conforme Pardos (1980) e Capitani (1982). Essa mistura pode interferir na eficiência do ethephon que também requer um $\mathrm{pH}$ maior que 3,5 para que o ácido 2-cloroetil fosfônico libere o etileno junto ao tecido vegetal.

A aplicação da pasta estimulante foi efetuada logo após a remoção do alburno e, para se obter uma máxima produção, a aplicação foi uniforme em toda a face removida, fazendo com que a solução aplicada penetrasse nos canais resiníferos.

Estas pastas foram aplicadas em intervalos de 15 dias, sendo que a quantidade de resina obtida nas 5 estrias foram coletadas e pesadas através de uma balança de mão (dinamômetro), a cada 2 meses, aproximadamente. O dinamômetro tem sido utilizado convencionalmente na pesagem das 
amostras, sendo que, para efeito comparativo com outros ensaios, foi aplicada a mesma metodologia.

A resina amostrada para análise de breu, terebintina, água e impurezas, acidez, alfa e beta-pineno, foi coletada do bloco 11, composto de 30 árvores, 5 tratamentos e controle. A coleta foi realizada separando-se uma porção de resina de cada árvore sob o mesmo tratamento, formando-se assim uma única amostra homogênea.

As resinas foram submetidas a um processo de destilação, separando-se o breu da terebintina, os quais foram devidamente quantificados e analisados quanto à percentagem de breu, terebintina, água e impurezas, além de acidez.

A terebintina foi analisada quanto a sua composição química, para esta determinação utilizou-se:

- cromatógrafo de fase gasosa, CG17 modelo 5890 séries II;

- detector - ionização de chama;

- coluna - sílica, 0,6 x $200 \mathrm{~cm}$ de aço inox, empacotado com DEGS a 18\%, modelo HP $20 \mathrm{M}$ carbowax;

- gás de arraste - hélio;

- temperatura da coluna $70^{\circ} \mathrm{C}$;

- injeção de amostra - 1 microlitro.

A água e impurezas foram determinadas por diferença; sendo que o número de acidez foi estabelecido pela fórmula apropriada (CLEMENTS, 1960).

\section{RESULTADOS E DISCUSSÃO}

$\mathrm{Na} 1^{\mathrm{a}}$ pesagem, observou-se que a Pasta Vermelha Eldorado, Pasta Vermelha Alchem e Pasta Preta Resinas Brasil aumentaram a produção de resina com relação ao Controle (Pasta Preta Planebrás). Já a Pasta Teste (paraquat + ethephon) e a Pasta com Ethephon Planebrás não diferiram do Controle.

Notou-se que, na $2^{\mathrm{a}}$ pesagem, a Pasta Teste (Paraquat + Ethephon) mostrou-se inferior em relação à Pasta Vermelha Alchem, Pasta com Ethephon Planebrás, Pasta Preta Resinas Brasil e Pasta Vermelha Eldorado. Na $3^{\text {a }}$ pesagem, a Pasta Teste (Paraquat + Ethephon) levou a produções inferiores aos tratamentos com Pasta Vermelha Alchem e Pasta Vermelha Eldorado, os quais não diferiram significativamente dos demais tratamentos. Já na $4^{\mathrm{a}}$ pesagem, a Pasta Teste (Paraquat + Ethephon), a Pasta Vermelha Alchem e a Pasta Preta Resinas Brasil se revelaram inferiores em relação ao Controle (Pasta Preta Planebrás), sendo que a Pasta com Ethephon
Planebrás e a Pasta Vermelha Eldorado não diferiram do Controle. Na $5^{\text {a }}$ pesagem, a Pasta Vermelha Eldorado mostrou-se superior em relação à Pasta Vermelha Alchem, já os demais tratamentos não diferiram do Controle.

$\mathrm{Na} 6^{\mathrm{a}}$ pesagem, a Pasta Vermelha Eldorado, a Pasta Preta Resinas Brasil e a Pasta Vermelha Alchem mostraram-se superiores ao Controle o qual não diferiu da Pasta Teste (Paraquat + Ethephon) e da Pasta com Ethephon Planebrás.

$\mathrm{Na} 7^{\mathrm{a}}$ pesagem, as Pasta Vermelha Alchem e Pasta Ethephon Planebrás mostraram-se superiores em relação à Pasta Teste (Paraquat + Ethephon). Na 8 pesagem, a Pasta Vermelha Alchem novamente revelou-se superior quando comparada com a Pasta Teste (Paraquat + Ethephon) sendo que essa não diferiu das demais.

$\mathrm{Na} 9^{\text {a }}$ pesagem, a Pasta Vermelha Alchem e a Pasta com Ethephon Planebrás mostraram-se superiores a Pasta Teste (Paraquat + Ethephon) e novamente, na $10^{a}$ pesagem, observou-se que a Pasta Vermelha Alchem, a Pasta Vermelha Eldorado e a Pasta com Ethephon Planebrás foram superiores à Pasta Teste (Paraquat + Ethephon), sendo que não diferiram do Controle e da Pasta Preta Resinas Brasil (Tabela 1).

Desses resultados, pode-se considerar que, somente nas $1^{\mathrm{a}}$ e $6^{\mathrm{a}}$ pesagens, a Pasta Vermelha Eldorado, Pasta Vermelha Alchem e a Pasta Preta Resinas Brasil resultaram nas maiores produções de resina em relação ao Controle (Pasta Preta Planebrás). Pode-se também observar que a Pasta Teste (Paraquat + Ethephon) não levou a incrementos de produção em relação ao Controle durante todo o experimento (Tabela 1).

A Tabela 2 apresenta os resultados percentuais de breu, terebintina, água e impurezas além da acidez das resinas após o processo de destilação.

A resina das árvores tratadas com Pasta com Ethephon Planebrás apresentou a maior porcentagem de breu e terebintina com relação às demais pastas estimulantes e, consequentemente, a menor porcentagem de água e impurezas. A resina obtida das árvores Controle (Pasta Preta Planebrás) tendeu a apresentar o maior número de acidez quando comparado às demais pastas estimulantes. Brito et al. (1978) e Capitani et al. (1980) verificaram valores de breu superiores aos do presente experimento, sendo que os níveis de terebintina revelaram-se semelhantes. Coraiola (1978) citou a composição média da resina recebida na usina com breu $78 \%$, terebintina $17 \%$ e água e impurezas $5 \%$. Esses va- 
TABELA 1: Pesos de resina de Pinus elliottii var. elliottii coletadas a cada 3 meses, resultantes de cinco estrias, em dez épocas de pesagens, durante 2 anos, resinado anteriormente com Pasta Vermelha.

TABLE 1: Weights of Pinus elliottii var. elliottii resin collected every 3 months, resulting streaks of five, ten times weighing over 2 years, previously with Resin Red Paste.

\begin{tabular}{cccccc}
\hline \multirow{2}{*}{ Tratamento } & \multicolumn{5}{c}{ Peso de Resina $(\mathrm{g})$} \\
\cline { 2 - 6 } & $1^{\mathrm{a}}$ Pesagem & $2^{\mathrm{a}}$ Pesagem & $3^{\mathrm{a}}$ Pesagem & $4^{\mathrm{a}}$ Pesagem & $5^{\mathrm{a}}$ Pesagem \\
\hline Controle & $467,13 \mathrm{c}$ & $528,36 \mathrm{ab}$ & $502,11 \mathrm{ab}$ & $781,11 \mathrm{a}$ & $556,69 \mathrm{ab}$ \\
Pasta A & $610,86 \mathrm{ab}$ & $614,14 \mathrm{a}$ & $548,94 \mathrm{a}$ & $507,78 \mathrm{~b}$ & $429,17 \mathrm{~b}$ \\
Pasta B & $527,41 \mathrm{bc}$ & $584,94 \mathrm{a}$ & $512,78 \mathrm{ab}$ & $615,83 \mathrm{ab}$ & $531,25 \mathrm{ab}$ \\
Pasta C & $583,47 \mathrm{ab}$ & $649,53 \mathrm{a}$ & $518,38 \mathrm{ab}$ & $500,66 \mathrm{~b}$ & $519,67 \mathrm{ab}$ \\
Pasta D & $668,19 \mathrm{a}$ & $634,47 \mathrm{a}$ & $533,00 \mathrm{a}$ & $581,66 \mathrm{ab}$ & $601,39 \mathrm{a}$ \\
Pasta Teste & $457,67 \mathrm{c}$ & $361,27 \mathrm{~b}$ & $410,33 \mathrm{~b}$ & $519,33 \mathrm{~b}$ & $504,33 \mathrm{ab}$ \\
F (Trat) & $9,65^{\text {n.s. }}$ & $7,75^{*}$ & $3,72^{*}$ & $3,29^{* *}$ & $3,45^{* *}$ \\
C.V. $(\%)$ & 11,84 & 17,19 & 12,28 & 24,60 & 14,45 \\
\hline
\end{tabular}

\begin{tabular}{cccccc}
\hline \multirow{2}{*}{ Tratamento } & \multicolumn{5}{c}{ Peso de Resina $(\mathrm{g})$} \\
\cline { 2 - 6 } & $6^{\mathrm{a}}$ Pesagem & $7^{\mathrm{a}}$ Pesagem & $8^{\mathrm{a}}$ Pesagem & $9^{\mathrm{a}}$ Pesagem & $10^{\mathrm{a}}$ Pesagem \\
\hline Controle & $591,84 \mathrm{bc}$ & $611,89 \mathrm{ab}$ & $514,72 \mathrm{ab}$ & $430,27 \mathrm{ab}$ & $549,67 \mathrm{ab}$ \\
Pasta A & $774,50 \mathrm{a}$ & $726,50 \mathrm{a}$ & $594,28 \mathrm{a}$ & $462,55 \mathrm{a}$ & $592,61 \mathrm{a}$ \\
Pasta B & $704,83 \mathrm{ab}$ & $673,27 \mathrm{a}$ & $531,11 \mathrm{ab}$ & $475,77 \mathrm{a}$ & $577,66 \mathrm{a}$ \\
Pasta C & $785,83 \mathrm{a}$ & $644,16 \mathrm{ab}$ & $517,61 \mathrm{ab}$ & $420,67 \mathrm{ab}$ & $548,72 \mathrm{ab}$ \\
Pasta D & $825,00 \mathrm{a}$ & $627,44 \mathrm{ab}$ & $516,27 \mathrm{ab}$ & $440,77 \mathrm{ab}$ & $588,27 \mathrm{a}$ \\
Pasta Teste & $499,00 \mathrm{c}$ & $502,00 \mathrm{~b}$ & $436,05 \mathrm{~b}$ & $359,33 \mathrm{~b}$ & $432,61 \mathrm{~b}$ \\
F (Trat) & $10,09^{*}$ & $4,51^{*}$ & $4,27^{*}$ & $2,98^{*}$ & $3,69^{* *}$ \\
C.V. (\%) & 14,04 & 13,67 & 11,52 & 13,41 & 13,88 \\
\hline
\end{tabular}

Em que: Médias seguidas de letras distintas, dentro de uma mesma coluna, diferem pelo teste de Tukey ao nível de $5 \%$ de probabilidade. $* *$ Significativo ao nível de $1 \%$ de probabilidade pelo teste $\mathrm{F}$. $*$ Significativo ao nível de $5 \%$ de probabilidade pelo teste F. ${ }^{\text {n.s. }}=$ não significativo. Controle: Pasta Planebrás; Pasta A: Pasta Vermelha Alchem; Pasta B: Pasta com Ethephon Planebrás; Pasta C: Pasta Preta Resinas Brasil; Pasta D: Pasta Vermelha Eldorado; Pasta Teste: Pasta Teste (Paraquat + Ethephon).

lores de breu e terebintina mostraram-se acima dos obtidos nesse trabalho; sendo que o de água e impurezas revelou-se inferior.

Aplicação de Pasta Preta Resinas Brasil levou a produção de menor porcentagem de breu na resina, sendo que a Pasta Teste (Paraquat + Ethephon) produziu a menor porcentagem de terebintina.

Quanto ao número de acidez, Brito et al. (1978) encontraram em Pinus elliottii var. elliottii o valor de 158,3 no primeiro trimestre e de 160,0 no segundo trimestre, sendo que para os tratamentos com diferentes pastas encontraram-se valores de 161,7 até 191,3 .

$\mathrm{Na}$ análise de terebintina, foi realizada a determinação do teor de alfa e beta-pineno (Tabela 3).

Considerando os padrões de alfa-pineno da ordem de $94,52 \%$ e de beta-pineno de $94,16 \%$, os resultados da Tabela 3 revelaram maior porcentagem de alfa-pineno $(53,31 \%)$ e menor de beta-pineno $(34,34 \%)$ nas amostras oriundas do Controle (Pasta Preta Planebrás), sendo que a Pasta Teste (Paraquat + Ethephon) apresentou uma menor porcentagem de alfa-pineno $(37,70 \%)$ e maior de beta-pineno $(50,41 \%)$. Brito et al. (1978) obtiveram valor de alfa-pineno da ordem de 63,00\% em Pinus elliottii var. elliottii, superior ao encontrado nessa espécie sob diferentes pastas estimulantes, no presente trabalho. Também observaram valor de beta-pineno de $26,30 \%$ e, portanto, inferior àqueles obtidos nesse experimento.

Coraiola (1978) obteve valor de 48,50\% para alfa-pineno e de $41,00 \%$ para beta-pineno, valores esses também encontrados nestas determinações. Capitani et al. (1980) encontraram valor superior de alfa-pineno, e muito inferior de beta-pineno quando comparado a este trabalho. 
TABELA 2: Resultados das análises de breu, terebintina e acidez da resina de Pinus elliottii var. elliottii, resultante da aplicação de cinco pastas estimulantes em relação ao Controle (Pasta Preta Planebrás).

TABLE 2: Results of analysis of pitch, turpentine and resin acid from Pinus elliottii var. elliottii, resulting from the application of five stimulant pastes compared to control (Black Paste Planebrás).

\begin{tabular}{ccccc}
\hline Tratamento & \% Breu & \% Terebintina & \% Água e Impurezas & $\mathrm{N}^{\circ}$ de Acidez \\
\hline Controle & 76,31 & 13,36 & 10,32 & 191,350 \\
Pasta A & 73,87 & 13,22 & 12,91 & 175,421 \\
Pasta B & 76,56 & 13,78 & 9,66 & 161,752 \\
Pasta C & 71,33 & 12,92 & 15,75 & 166,240 \\
Pasta D & 76,21 & 13,48 & 10,31 & 165,689 \\
Pasta TESTE & 75,49 & 10,37 & 14,14 & 167,325 \\
\hline
\end{tabular}

TABELA 3: Teores de alfa e beta-pineno apresentados pela terebintina da resina de Pinus elliottii var. elliottii resultante da aplicação de cinco pastas estimulantes em relação ao Controle (Pasta Preta Planebrás).

TABLE 3: Yields of alpha and beta-pinene of turpentine from the resin of Pinus elliottii var. elliottii, resulting from the application of five stimulant pastes compared to control (Black PastePlanebrás).

\begin{tabular}{ccc}
\hline Tratamento & Alfa-pineno (\%) & Beta-pineno (\%) \\
\hline Controle & 53,31 & 34,34 \\
Pasta A & 42,55 & 43,80 \\
Pasta B & 50,49 & 42,57 \\
Pasta C & 46,16 & 44,47 \\
Pasta D & 46,96 & 43,77 \\
Pasta TESTE & 37,70 & 50,41 \\
\hline
\end{tabular}

Os valores de alfa-pineno na terebintina mostram-se mais elevados no Controle (Pasta Preta Planebrás) e menores na Pasta Teste (Paraquat + Ethephon).

\section{CONCLUSÕES}

No estabelecimento da maior frequência de árvores de Pinus elliottii var. elliottii em plantio localizado no Horto florestal de Itatinga da ESALQ/USP, observou-se que as mesmas eram árvores de produção de resina intermediária entre dois extremos.

Verificou-se, durante 2,5 anos de explotação de resina, que as pastas estimulantes aplicadas diferiram nas 10 pesagens realizadas.

Infelizmente, pode-se considerar que a
Pasta Teste (Paraquat + Ethephon) não substituíu a ação do ácido sulfúrico no processo de produção de resina, sendo este mais relevante do que o efeito do ethephon nesse processo.

Nos tratamentos efetuados, a porcentagem de breu variou de $71,33 \%$ a $76,56 \%$. A terebintina oscilou de $10,37 \%$ a $13,78 \%$. O alfa-pineno variou de $37,70 \%$ a $53,31 \%$, sendo que o beta-pineno oscilou de $34,34 \%$ a $50,41 \%$.

\section{REFERÊNCIAS BIBLIOGRÁFICAS}

ALVIM, P. T. Base fisiológica da produção vegetal: curso intensivo de fisiologia vegetal. Itabuna, 1969. 49 p.

BRITO, J. O. et al. Resinagem e qualidade de resinas de pinheiros tropicais - comparações entre espécies e época de resinagem. Circular Técnica IPEF, Piracicaba, n. 35, p. 1-20, 1978.

BUCCI, L. A.; RIBAS, C.; ASSINI, J. L. Produção de resina de Pinus elliottii em Campos do Jordão. Boletim Técnico do Instituto Florestal, São Paulo, n. 2, p. 576-583, 1986.

CAPITANI, L. R. Primeiros resultados do uso de ethrel na resinagem de Pinus caribaeae var. hondurensis na reflorestadora Sacramento Resa Ltda. Circular Técnica IPEF, Piracicaba, n. 147, p. 1-5, 1982.

CAPITANI, L. R. et al. A potencialidade de resinagem de quatro espécies de Pinus tropicais na região de Sacramento, MG. Circular Técnica IPEF, Piracicaba, n. 110, p. 1-15, 1980.

CLEMENTS, R. W. Modern gum naval store methods. Washington, USDA, 1960. 29 p.

CORAIOLA, R. Obtenção e utilização da cola breu para a fabricação de papel. Boletim Técnico do Instituto Florestal, São Paulo, 1978. 16 p.

FERNANDES, P. S.; OSORIO, R. G. S. Efeito 
exaustivo de estimulantes químicos na resinagem intensiva de Pinus elliottii var. elliottii, Circular Técnica IPEF, Piracicaba, n. 41, p. 139-147, 1988. GARRIDO, M. A. O. Pesquisa sobre resinagem no Instituto Florestal. Silvicultura, São Paulo, v. 8, p. 43-45, 1983.

JOYCE, E. R.; DREW, J. S. C. M. Field experiment with paraquat substitutes. In: Annual Lightwood Research Conference, Atlanta, p.82-103, 1979. KRAMER, P. J.; KOZLOWSKI, T. T. Fisiologia das árvores. Lisboa: Fundação Calouste Gulbenkian, 1972. $745 \mathrm{p}$.

PARDOS, J. A. Influência de 2,4-D em la formación de canales resiniferos y en el crescimiento radial del xilema en plantitas de Pinus pinaster, Ait. Annales INIA Serie Recursos Naturales, Chile, n. 4, p. 157163, 1980.

PETERS, W. J.; ROBERTS, D. R. Ethrel bipyridylium synergism in flash pine. In: Ligthwood Research Coordinating Council. Jacksonville, 1977. p. 78-83. 